\title{
L'église Saint-Martin de Domprel (Doubs). Vestiges de la première église et de sépultures
}

David Billoin et avec la collaboration de Thierry Euvrard

\section{OpenEdition}

1 Journals

Édition électronique

URL : https://journals.openedition.org/cem/12302

DOI : $10.4000 /$ cem. 12302

ISSN : 1954-3093

Éditeur

Centre d'études médiévales Saint-Germain d'Auxerre

Référence électronique

David Billoin et avec la collaboration de Thierry Euvrard, «L'église Saint-Martin de Domprel (Doubs). Vestiges de la première église et de sépultures », Bulletin du centre d'études médiévales d'Auxerre I BUCEMA [En ligne], 16 | 2012, mis en ligne le 05 décembre 2012, consulté le 22 septembre 2022. URL http://journals.openedition.org/cem/12302 ; DOI : https://doi.org/10.4000/cem.12302

Ce document a été généré automatiquement le 22 septembre 2022.

Creative Commons - Attribution - Pas d'Utilisation Commerciale - Partage dans les Mêmes Conditions 4.0 International - CC BY-NC-SA 4.0

https://creativecommons.org/licenses/by-nc-sa/4.0/ 


\title{
L'église Saint-Martin de Domprel (Doubs). Vestiges de la première église et de sépultures
}

\author{
David Billoin et avec la collaboration de Thierry Euvrard
}

1 L'extension du cimetière de Domprel, commune du Doubs située sur le plateau de Vercel et de Valdahon, à $41 \mathrm{~km}$ à l'est de Besançon, a donné l'occasion d'effectuer un diagnostic archéologique à l'emplacement de l'église Saint-Martin aujourd'hui disparue ${ }^{1}$. Hormis son emplacement remarquable, en bordure d'une falaise sur le point culminant d'une colline $(790 \mathrm{~m})$ offrant un large panorama

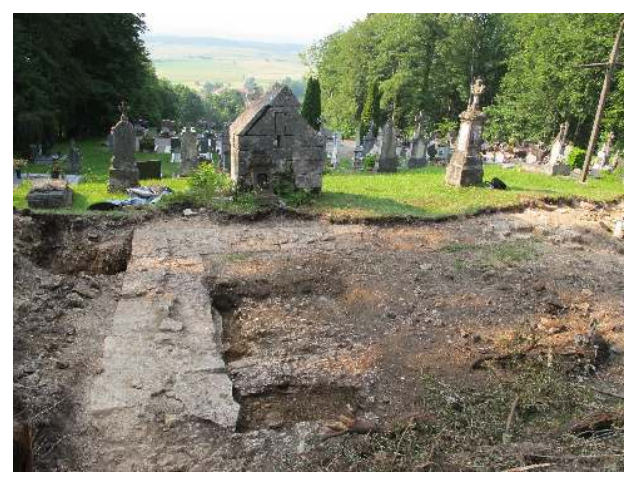
sur le paysage alentour, cette église est restée des plus méconnues, puisqu'elle n'apparaît dans les textes qu'en filigrane à l'occasion de correspondances et de suppliques justifiant son transfert. On ignore donc pratiquement tout de cet édifice cultuel et, notamment, de son origine. Du point de vue iconographique, elle n'apparaît que sur un plan aquarellé daté de 1743 retrouvé dans des archives privées.

2 L'histoire de cet édifice cultuel se confond avec celle du village, dans un secteur d'altitude réputé colonisé lors des grands défrichements monastiques des $\mathrm{X}^{\mathrm{e}}-\mathrm{XII}{ }^{\mathrm{e}}$ siècles. De fait, aucun témoignage archéologique plus précoce n'est enregistré sur le territoire communal, hormis le passage d'une voie d'époque indéterminée au toponyme $L a$ Pérouse. 


\section{État des connaissances}

3 L'existence du village est attestée au XII ${ }^{\mathrm{e}}$ siècle par la présence de la famille de Domprel, ancienne maison du comté de Bourgogne. Un Pierre de Domprel dirigeait l'abbaye de Montbenoit en 1139. La localité reçoit le nom de Dompré en 1264, Domprey en 1290. Le fief de Domprel relevait de la seigneurie de Passavant, puis passa sous la mouvance des comtes de Montbéliard-Würtemberg en $1407^{2}$.

4 L'église primitive de Domprel, sous l'invocation de saint Martin, antérieure au $\mathrm{XV}^{\mathrm{e}}$ siècle, était bâtie en arrière du cimetière au sommet de la colline du Bois de la Côte, surplombant le village (712 $\mathrm{m}$ d'altitude). Elle dépendait d'Eysson, mais les habitants de Domprel la considéraient comme paroissiale, ce qui opposa les deux communes de la fin du XVII à la fin du XVIII ${ }^{e}$ siècle. Abandonnée, cette église est reconstruite au centre du village. Les travaux commencent en 1765 et s'achèvent en 1786. De style gréco-romain et en forme de croix latine, la nef de cette nouvelle église comporte un pavage incluant deux tombes de prêtres, datées de 1635 et 1699 , rapportées de l'ancienne église, ainsi qu'une autre tombe dans le chœur. Les fragments d'une statue équestre de saint Martin passent pour avoir été trouvés à l'emplacement de l'ancienne église ${ }^{3}$, mais on ignore où ils sont conservés aujourd'hui.

5 Les documents concernant cette église Saint-Martin sont peu diserts. Elle n'apparaît dans les textes qu'au XvII ${ }^{\mathrm{e}}$ siècle lors de correspondances concernant sa reconstruction au centre du village ${ }^{4}$. Les raisons de ce transfert et quelques informations apparaissent à la lecture de ces lettres afin d'appuyer la demande, mais sans détailler l'aspect architectural de l'édifice. On apprend ainsi dans une supplique adressée au duc de Wurtemberg, prince souverain de Montbéliard et autres lieux, datée du 16 février 1770 :

«Qu'ils ont pris la résolution de rebâtir leur église qui étoit fort caduque et ruineuse placée d'ailleurs dans une forêt éloignée des deux villages et exposée à des profanations et vols continuels commis (...) ils constatent de son état ruineux (...). L'état caduque qui a été reconnu de l'ancienne église et surtout du coeur (...) et aux besoins pressants où ils étoient de rétablir cet édifice, dont l'emplacement leur étoit fort pénible, dans un endroit montueux et inaccessible pour les gens d'un certain âge (...).»

6 Dans une requête du 9 avril 1717, d'autres arguments sont exposés afin de justifier le déplacement de l'église :

«(...) L'église de Domprel est éloignée de la Sommette d'une bonne heure, que le chemin est très difficile en hyver qui dure six mois, tant à cause des neiges que des vents qui règnent dans cet endroit et qui rendent le chemin difficile et presque impraticable de temps d'hyver sans même avoir égard aux glaces qui sont ordinairement pendant l'hyver dans le chemin de ladite église estant bastie dans le bois au dessus de la colline ce qui prive de tous secours et des moyens de se chauffer ou rafraîchir après leur grande fatigue du chemin (...). »

7 Un seul plan aquarellé de 1743 (fig. 1) représente l'église de Domprel sur la ligne de crête boisée, sous la forme d'un bâtiment rectangulaire, où une ouverture est visible, couvert d'une toiture débordante, vraisemblablement à trois pans, surmontée à l'ouest par un clocher muni d'une baie cintrée, couvert d'une toiture à quatre pas et d'une croix. Un chemin monte légèrement à l'oblique en direction de l'édifice, évitant ainsi le front de falaise à l'aplomb de ce côté de la colline. 
Fig. 1 - La seule représentation connue de l'église Saint-Martin, extrait d'un plan aquarellé de 1743 (coll. particulière ; cl. P. Haut).

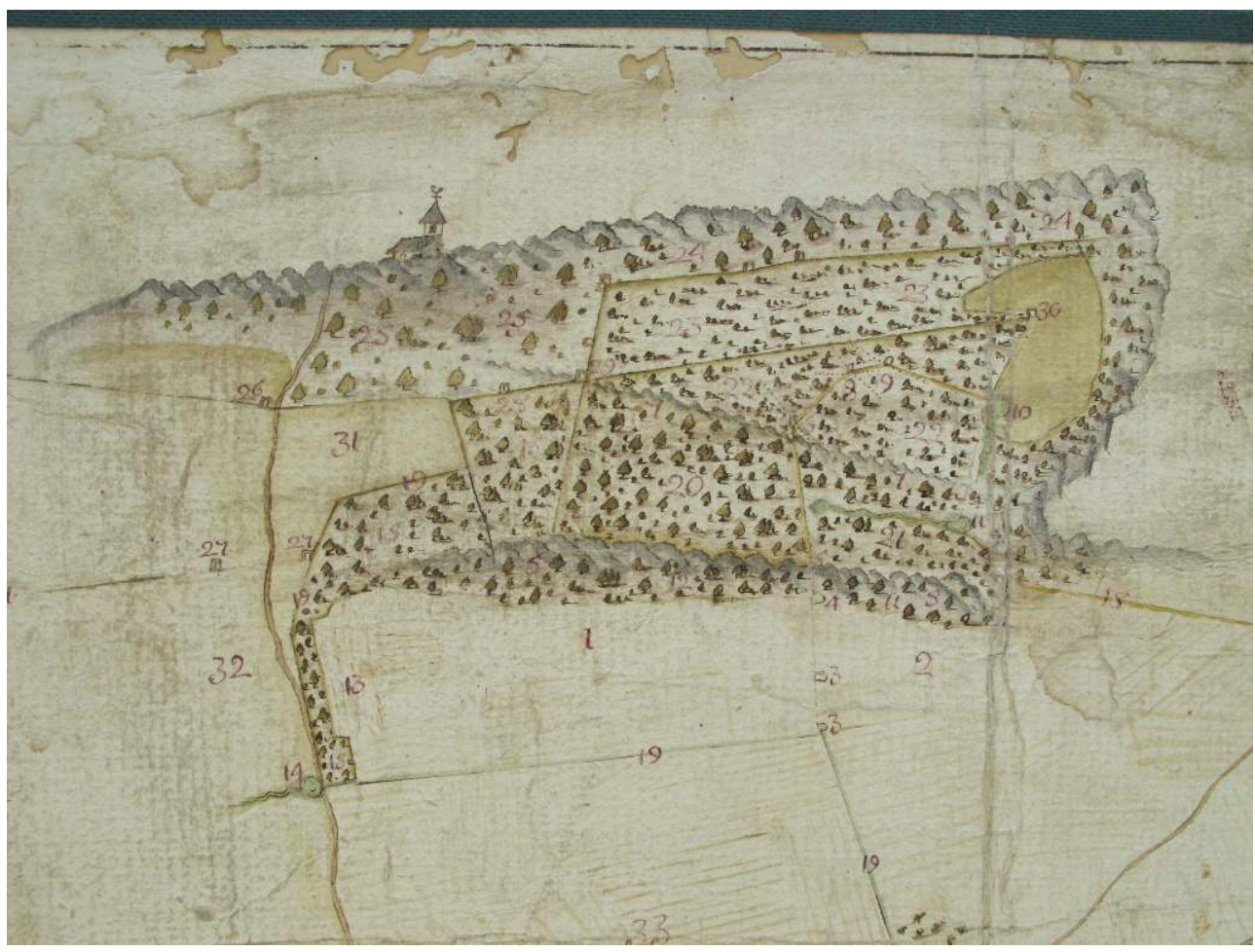

\section{L'église}

Trois sondages, totalisant près de $100 \mathrm{~m}^{2}$ ouverts, permettent de reconnaître les vestiges de cet édifice apparaissant immédiatement sous la terre végétale (fig. 2). 
Fig. 2 - Plan général des vestiges de l'église et des sépultures (D. Billoin et P. Haut).

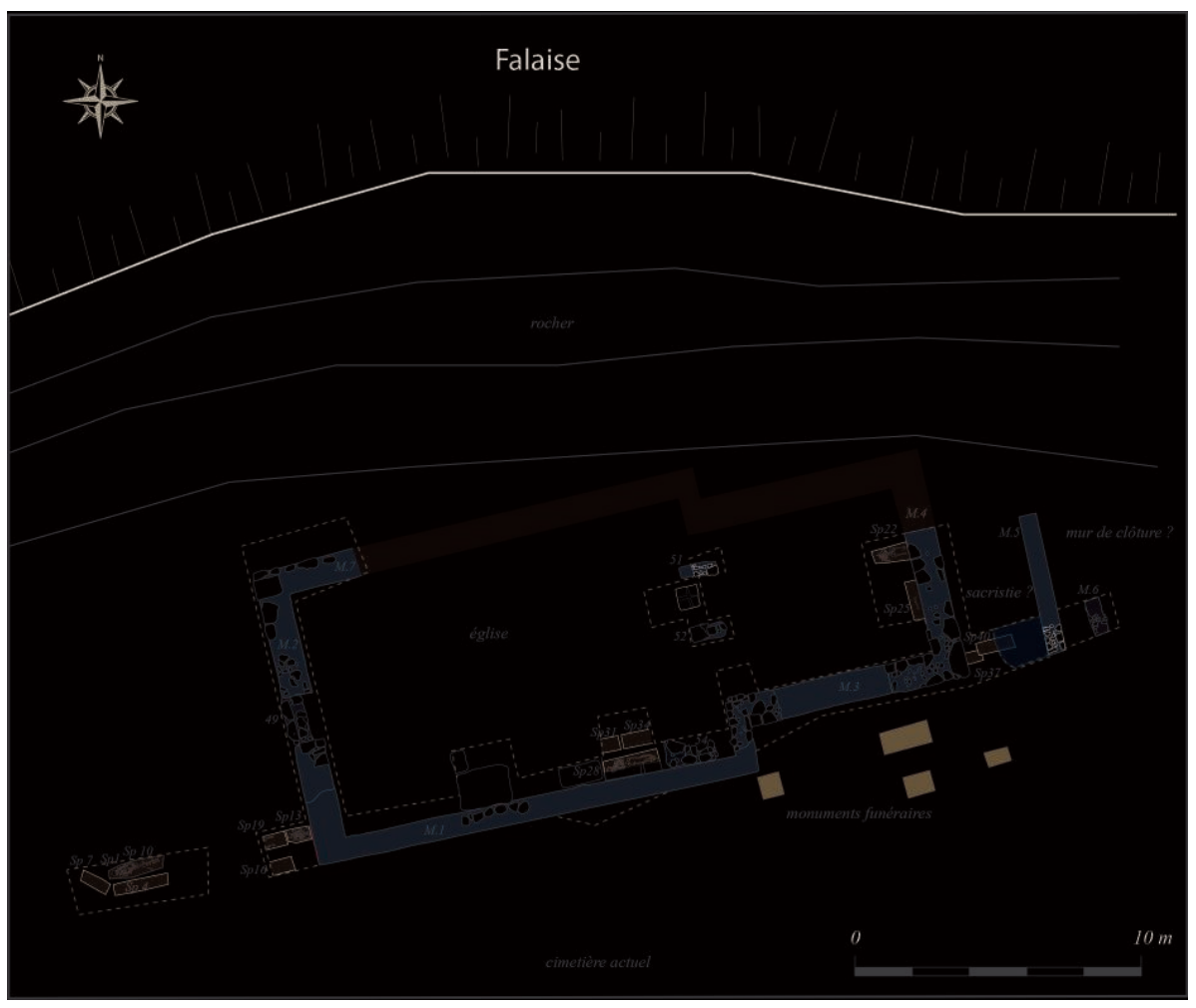

9 Toute la façade orientale a été mise au jour, affichant un mur gouttereau M1 de $15 \mathrm{~m}$ de longueur, prolongé d'un chevet débordant M3 de 7,5 m de long, soit un édifice d'une longueur totale de $22,50 \mathrm{~m}$. Les tranchées perpendiculaires ont permis de reconnaître son plan et, en particulier, la largeur complète du portail M2, de $10 \mathrm{~m}$, muni d'une entrée centrée de 1,60 m de largeur, disposant encore de marches et d'un seuil ; ainsi que le chevet plat M4, suivi sur une longueur de $5 \mathrm{~m}$ sur les 7,5 m de largeur restituable. Il s'agit donc d'un édifice rectangulaire à chevet plat débordant d'une certaine ampleur et occupant pratiquement la totalité de l'espace compris entre la limite du cimetière actuel et la ligne de crête marquée par la falaise au nord. Cette position topographique dominante est remarquable, avec un large point de vue, tant au nord que vers le sud, et ancre l'église dans le paysage environnant (fig. 3). 
Fig. 3 - Le mur du chevet et les sépultures recoupées par sa construction (P. Haut).

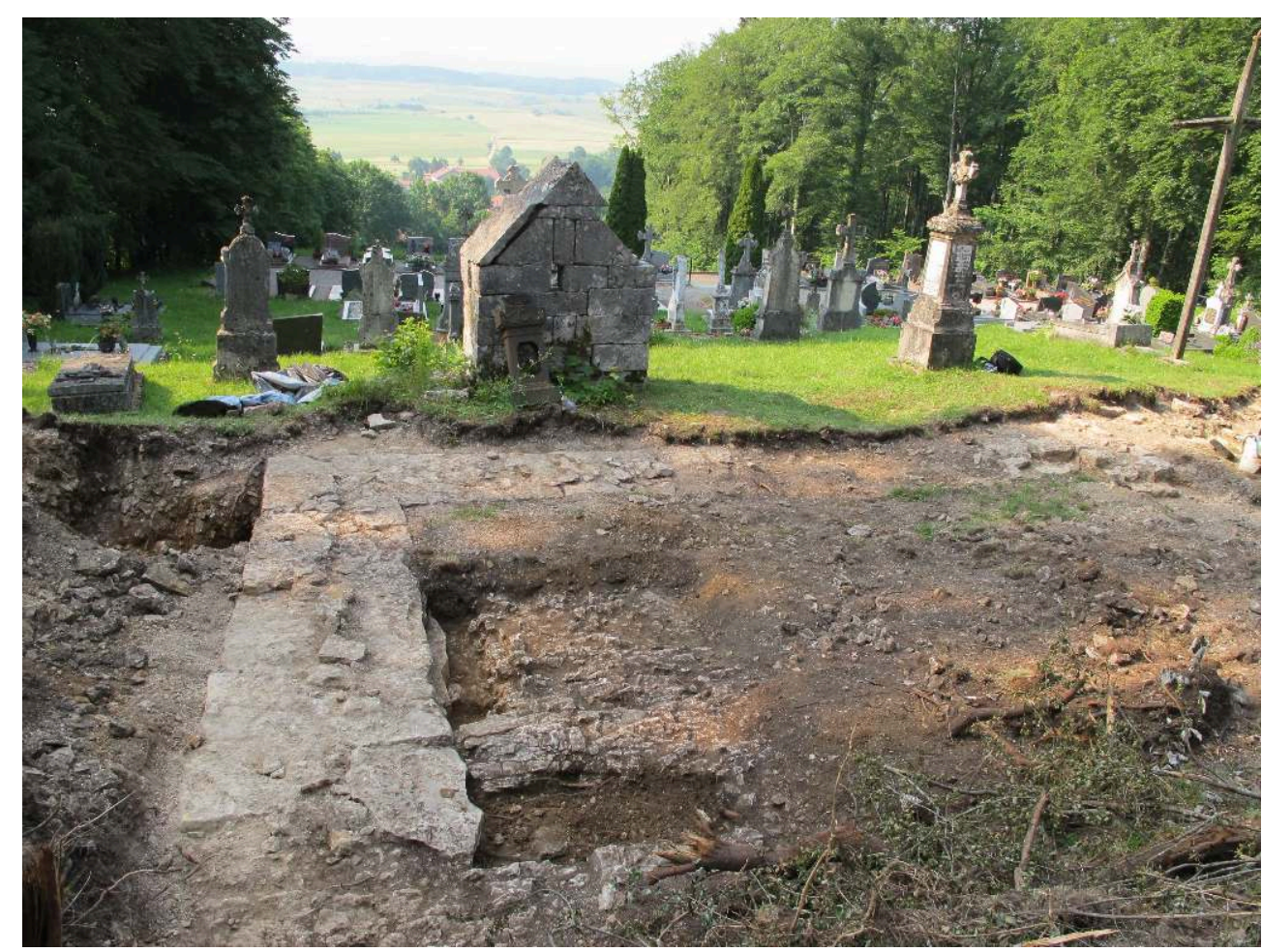

Larges de 0,90 m, les murs gouttereaux sont conservés en élévation - davantage dans la partie nord - sur une à trois assises de gros moellons de calcaire local en appareil de revêtement liés à un mortier blanc graveleux, sur une fondation large de $1,10 \mathrm{~m}$, constituée de gros moellons et de blocs de roche mis en forme posant directement sur la roche. Le chevet est composé de fondations de murs d'une largeur oscillant entre 1 et 1,05 m, s'accolant à ceux de l'église (M3 et M4). Ils sont constitués de gros moellons calcaires et de blocs de roche mis en forme en deux appareils de revêtement, liés à un mortier sableux de couleur crème. De grandes dalles quadrangulaires usées en calcaire subsistent dans la nef, en particulier en bordure intérieure du mur M1 et à la jonction avec le chœur. Elles constituent le pavage intérieur de l'église, mais, à l'évidence, certaines d'entre elles manquent et ont été récupérées, à l'exemple des pierres tombales de prêtres transférées dans la nouvelle église. Cependant, un fragment de l'une des dalles, entre les deux piles du chœur, est orné d'une croix fleurdelisée gravée (fig. 4). Ces bases de piles quadrangulaires $(0,90 \times 1,20 \mathrm{~m})$, construites à la hauteur du mur d'épaulement de la nef, correspondent vraisemblablement à un système de voutement séparant la nef et le chœur de l'église, selon trois arcades distribuant un espace d'environ 1,65 $\mathrm{m}$ de large chacun. 
Fig. 4 - Fragment de dalle de sol orné d'une croix fleurdelisée à l'extrémité de la nef et entre les deux piles du chœur (P. Haut).

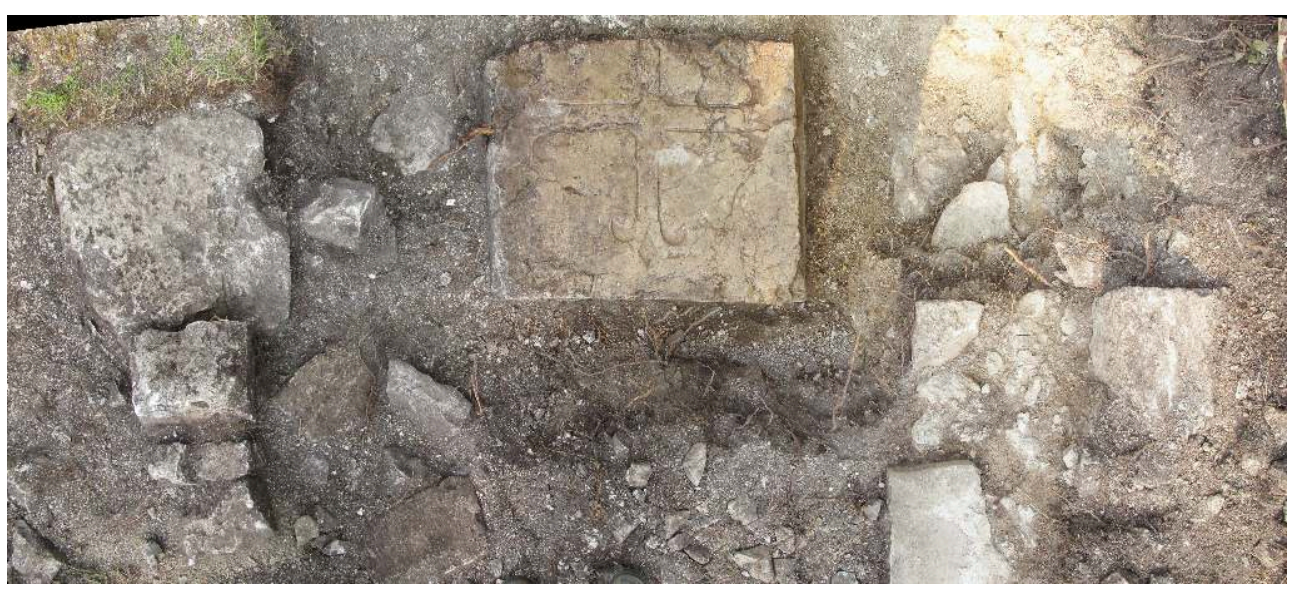

L'entrée de l'édifice est marquée par une interruption du mur M2, large de 1,60 m, centré sur le portail et l'aménagement d'un seuil formant une marche et une dalle de sol à l'intérieur de l'église à $0,10 \mathrm{~m}$ en contrebas. Ces deux pierres portent des traces d'usure bien marquées par une circulation fréquente et une petite croix est gravée sur l'une d'entre elle. Cette entrée marque l'axe de la nef centrale, mais affiche un décalage par rapport à l'axe des piles du chœur (contrainte topographique ?). Les fondations d'un autel se signalent par un massif de maçonnerie accolé au mur gouttereau sud, un peu en retrait du mur d'épaulement de la nef de l'église (fig. 2, n 54 ). De forme quadrangulaire, il affiche des dimensions de l'ordre de 1 x 1,75 m et est constitué d'un blocage de pierres noyées dans du mortier sableux de couleur jaune.

Deux murs parallèles M5 et M6 sont rencontrés à l'est du chevet. Distant de 2,75 m du chevet, le premier, suivi sur une longueur de $4,80 \mathrm{~m}$, est affleurant. Large de 0,65 m, il est composé de moellons calcaires de taille moyenne en appareil de revêtement, liés à un mortier sableux blanc. Sa face ouest est couverte d'un enduit également lissé sur $3 \mathrm{~cm}$ d'épaisseur. Cet enduit vient s'interrompre à la hauteur d'un sol en mortier dégagé sur une surface d'environ $2 \mathrm{~m}^{2}$, marquant l'emplacement d'un sol intérieur. Si la jonction avec le mur M4 du chevet n'est plus conservée, en revanche le remblai de démolition couvrant ce sol vient s'interrompre à son contact. Il est donc possible de restituer l'emprise de ce niveau intérieur entre ces deux murs, témoignant d'une construction accolée au chevet. Une couche d'occupation grisâtre couvre ce sol et a livré un lot de monnaies important pour une si petite surface, laissant supposer l'éparpillement d'une cache (peut-être une bourse) lors de la démolition de cette construction. Le parement est de ce mur montre des moellons bien assisés et bien taillés, destinés à être apparents sur l'extérieur de la construction. Le second mur M6 est similaire au mur M5, tant dans ses dimensions et son orientation que dans sa technique de construction. Il est composé de deux parements de moellons bien assisés et bien taillés qui évoquent un mur extérieur, à une distance d'un mètre du précédant, marquant peut-être la limite de cet espace cultuel.

\section{Les tombes antérieures à l'église}

Elles sont au nombre de cinq au minimum, d'après les recoupements observés entre elles et avec l'édifice (fig. 2 : SEP. 13, 19, 22, 25 et 37), mais peut-être davantage, 
certaines n'ayant aucun lien physique ou stratigraphique avec les murs du bâtiment. La tombe SEP. 13 est directement recoupée par les fondations de l'angle du portail et du mur gouttereau sud, à la hauteur de la partie supérieure des membres inférieurs (fig. 5).

Fig. 5 - Situation de la sépulture SEP. 30 recoupée par les fondations de l'angle du portail de l'église (P. Haut).

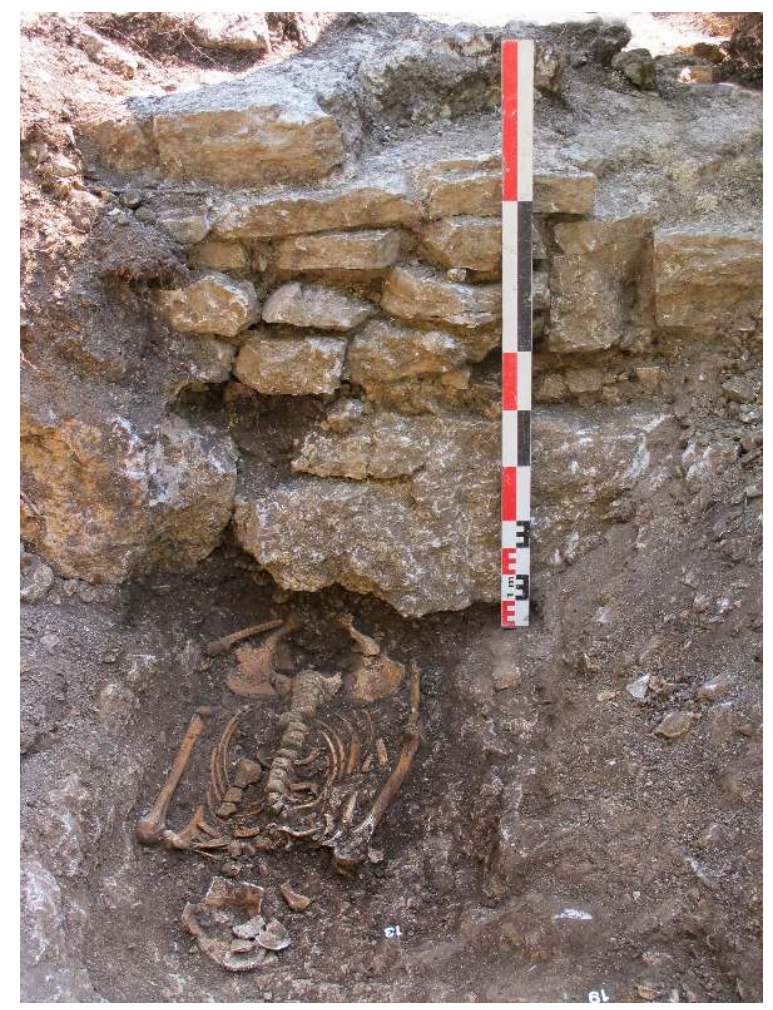

14 Apparaissant à une profondeur de $0,65 \mathrm{~m}$, son creusement forme une alvéole assez étroite dans la roche, à fond sensiblement plat. L'individu adulte est allongé sur le dos, les bras le long du corps. Quelques désordres mineurs sont observés, en relation directe avec les fondations, notamment le déplacement de l'avant-bras gauche. L'étroitesse de la tombe et la mise à plat du squelette suggèrent une inhumation en pleine terre. Cette sépulture recoupe la partie basse de la tombe SEP.16, de même orientation et qui atteint le socle rocheux. Voisine, la sépulture SEP. 19 est vraisemblablement contemporaine par son alignement avec les deux autres et une profondeur très similaire.

Trois autres sépultures (SEP. 22, 25 et 37) sont antérieures au chevet, comme l'atteste leur recoupement par le mur M4. Si les tombes SEP. 22 et 37 sont orientée est-ouest, la dernière a une orientation nord-sud (fig. 6). 
Fig. 6 - Le mur du chevet et les sépultures SEP. 22 et 25 recoupées par sa construction (P. Haut).

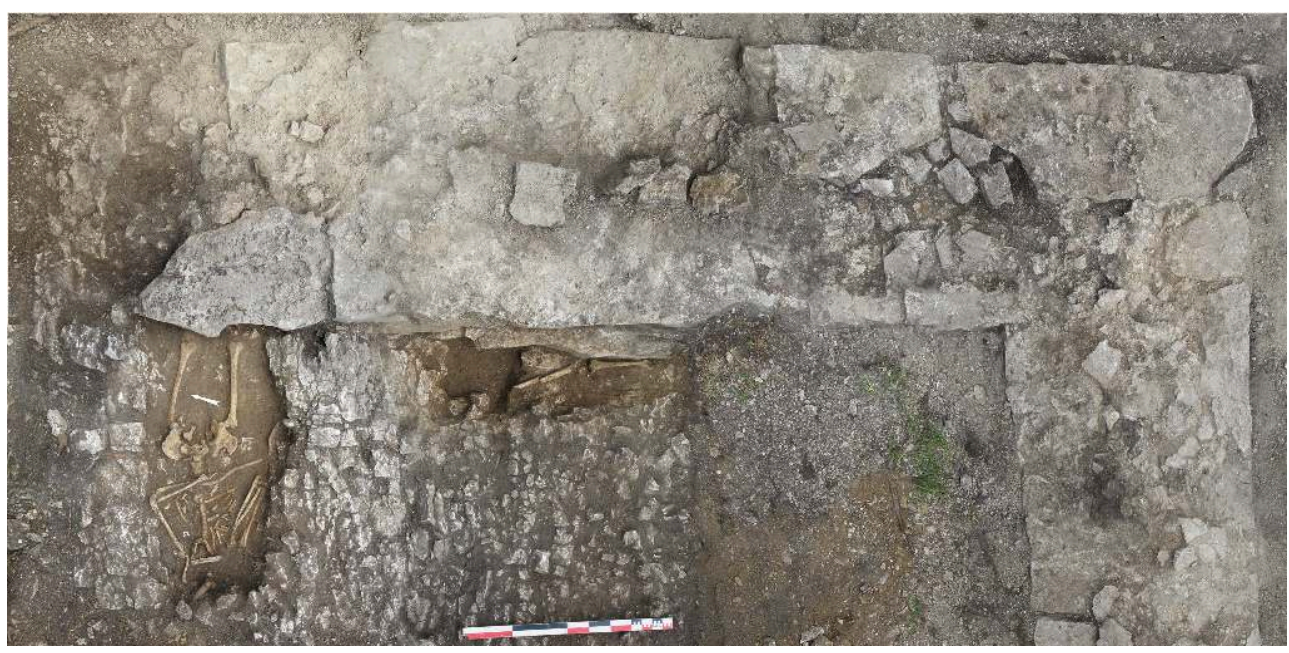

De l'individu de la tombe SEP. 37 ne subsistent que les membres inférieurs sur 0,40 m de longueur environ, cette tombe étant recoupée par une autre dans sa partie basse. Les fémurs appartiennent à un adulte ; le droit porte une excroissance osseuse importante, identifiée comme une dysplasie coxaux-fémorale, pathologie congénitale invalidante 5 .

\section{Les inhumations pratiquées dans l'église}

Seules les trois sépultures situées dans la nef, le long du mur gouttereau sud, sont assurément contemporaines de l'église (fig. $2:$ SEP. 28,31 et 34). Une seule d'entre elles a été fouillée (SEP. 28), les autres n'apparaissant que partiellement sous la forme de creusements rectangulaires, bien orientés est-ouest par rapport au mur gouttereau M1. Ces remplissages sont visibles - comme pour la tombe SEP. 28 - à la hauteur d'apparition des maçonneries, à une profondeur de $0,20 \mathrm{~m}$, à un endroit de la nef où les dalles de pavement du sol manquent. D'une profondeur de $0,60 \mathrm{~m}$, atteignant le substrat rocheux, la sépulture SEP. 28 révèle un jeune adulte de sexe vraisemblablement féminin, dont le corps est placé en décubitus dorsal, les membres supérieurs fléchis sur la poitrine et les membres inférieurs en extension. Sa tête, tournée vers le sud, est accolée au mur gouttereau, alors que l'articulation du bras gauche prend appui contre ce dernier. La mise à plat des os coxaux et du grill costal évoque une décomposition en espace vide, ainsi que la migration de la plupart des os du squelette. Seul sondage profond à l'intérieur de cet édifice, il révèle une densité de sépultures importante dans la nef.

Aucune de ces tombes n'a livré de mobilier funéraire, pas plus que leur comblement, et les architectures funéraires peu caractéristiques - inhumations en pleine terre et contenant boisé - n'apportent aucun argument chronologique. Prises dans leur ensemble, toutes ces tombes attestent l'existence d'un site funéraire préexistant à la construction de l'église, fonction qui se perpétue à l'intérieur comme à l'extérieur de cet édifice jusqu'à sa phase d'abandon. 


\section{Synthèse}

Le plan même de cet édifice rectangulaire à abside quadrangulaire charpentée est difficilement datable. Il est attesté dès le viI ${ }^{\mathrm{e}}$ siècle à l'exemple de l'église Saint-Pierre à Sainte-Ursanne (Jura Suisse) et au XII ${ }^{e}$ siècle, avec une voûte sur le cœur, comme sur l'église Notre-Dame de Saint-Claude (Jura), voire jusqu'aux XIII ${ }^{\mathrm{e}}-\mathrm{XIV}{ }^{\mathrm{e}}$ siècles. Un seul état est reconnu du point de vue des maçonneries et d'après les nombreuses monnaies recueillies, qui constituent les seuls éléments chronologiques pertinents. Leur éparpillement sur le sol de la sacristie lors de son effondrement, offre un bon terminus de cette phase d'abandon. Ce lot de vingt-sept monnaies est homogène et couvre le milieu du XVII ${ }^{\mathrm{e}}$ siècle. Celles retrouvées en différents endroits de la nef sont légèrement plus précoces et témoignent d'une occupation autour des $\mathrm{Xv}^{\mathrm{e}}-\mathrm{XVI} \mathrm{I}^{\mathrm{e}}$ siècles, bien qu'une monnaie plus récente, un cinq sol de l'atelier de Strasbourg, avec un millésime de 1704, puisse être liée à la récupération du dallage de l'église (fig. 7 et 8).

En définitive, seules les sépultures recoupées, tant par le portail que le chevet, illustrent une phase d'inhumations antérieure à la construction de cet édifice, sans toutefois apporter de précision en l'absence de mobilier et d'architecture funéraire spécifique. Des datations radiocarbones ont donc été engagées et offrent pour la sépulture 13 une période d'inhumation entre 1040 et 1213 apr. J.-C. (Ly-15452), avec un pic pour l'année 1158, et l'intervalle de 1051 à 1223 apr. J.-C. (Ly-14451) pour la sépulture 22, avec un pic sur l'année 1183.

Ces deux résultats attestent l'existence d'un premier cimetière dans la seconde moitié du XII ${ }^{e}$ siècle, précédant de peu l'édification de la première église. Cette période de fondation correspond à la période d'apparition du village dans les textes et sensiblement à ceux du plateau environnant, illustrant un peuplement assez tardif de ce secteur de moyenne montagne en relation avec la conquête de nouveaux terroirs. Ces défrichements traditionnels des $\mathrm{X}^{\mathrm{e}}$-XII ${ }^{\mathrm{e}}$ siècles, qui marquent la forêt comtoise, sont toutefois limités aux secteurs en marge des itinéraires antiques traversant le massif du Jura, qui se caractérisent par une occupation beaucoup plus précoce, même s'ils restent encore sous-documentés par l'archéologie. 
Fig. 7 - Catalogue des monnaies (T. Euvrard).

\begin{tabular}{|c|c|c|c|}
\hline$N^{0}$ & type de monnaie/atelier & & Localisation \\
\hline & Quinzain & Louis XIV & $\begin{array}{l}\text { coneur } \\
\text { civer }\end{array}$ \\
\hline 3 & Double denicr. Dole & Philippe IV frappé après |637 & coverr \\
\hline & indéterminés & & \\
\hline 7 & Angragne, Auxonne & $\begin{array}{l}\text { Charles le Téméraire } \\
(1467 / 1477)\end{array}$ & \\
\hline 8 & Angrogne, Auxonne & $\begin{array}{l}\text { Charles le Téméraire } \\
\text { (1467/1477) }\end{array}$ & nef \\
\hline y & Double tournois & Francois ler & nef \\
\hline 10 & Fünfer, Fribourg & XVle & \\
\hline$\frac{11}{12}$ & indélermine & & \\
\hline$\frac{12}{13}$ & $\begin{array}{l}\text { indéteminins } \\
\text { indétrmins }\end{array}$ & & \\
\hline 14 & Double toumois, Cuisery & Philippe Le Bon (1419:1467) & \\
\hline & Angrogne, Auxomie & Philippe le Ban (1419/1467) & \\
\hline \begin{tabular}{|l|}
16 \\
17
\end{tabular} & 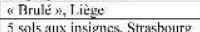 & $\frac{1419: 1455}{1704}$ & \begin{tabular}{|l} 
Exxlérieur du chevel \\
solnef
\end{tabular} \\
\hline 18 & Double Denier. Dole & Plhilippe IV frappé aprés 1637 & sacristic (?) \\
\hline & & & \\
\hline 20 & Carolus, Besançum & $\begin{array}{l}\text { Charles-Quimt } \\
\text { Daté } 16 \text { incomplet mais } \\
\text { début XVIIc }\end{array}$ & sacristie (?) \\
\hline 21 & Double tournois & Lowis XIII & sacristie (?) \\
\hline 22 & $\begin{array}{l}\text { Double tournois, Arches et } \\
\text { Charkcrille }\end{array}$ & Xville & sacristic (?) \\
\hline 23 & Double Denicr, Dole & $\begin{array}{l}\text { Philipps IV } \\
\text { frappe apres } 1637\end{array}$ & sacristic (?) \\
\hline 24 & Denicr, Dole & 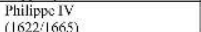 & sacristic (?) \\
\hline 25 & Deux denicrs, Dolc & $\begin{array}{l}\text { Plilippe II frappé cntre } 1591 \text { et } \\
1607\end{array}$ & sacristic ( ? \\
\hline 26 & Deux deniers Ier type. Dole & $\begin{array}{l}\text { Albert et Isabclle } \\
\text { Trappé enlre } 1608 \text { et } 1612\end{array}$ & sacristic (?) \\
\hline 27 & Deux Denier Ier type, Dole & $\begin{array}{l}\text { Albert ct Isabclle } \\
\text { Trappé entre } 1608 \text { et } 1612\end{array}$ & sacristic ( $?$ ) \\
\hline 28 & Denicr tournois, Dombes & & sacristic $(n)$ \\
\hline & inditerminis & & sacristic (n) \\
\hline $\begin{array}{l}30 \\
31\end{array}$ & $\begin{array}{l}\text { indeterminie } \\
\text { Deux deniers seconnd type, }\end{array}$ & & 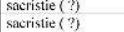 \\
\hline & $\begin{array}{l}\text { Dole } \\
\text { Don }\end{array}$ & 1612 et 1621 & \\
\hline & Double tournois & 1...nis XIII & sacrist \\
\hline & Double Lcumois, Sedan & & sacristie (?) \\
\hline 34 & Double toumois, Paris & 1613 & sacris \\
\hline & indḱterminé & & sacri \\
\hline$\frac{36}{37}$ & Double tournois, Paris & 1613 & sacristie (?) \\
\hline 38 & Dowhle Loumois, Paris & L.nuis XIIII & sacristie (?) \\
\hline 39 & 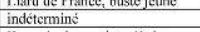 & & $\begin{array}{l}\text { satrisiter(?) } \\
\text { sacristic ? ? }\end{array}$ \\
\hline & Deux deniers points, Dole & Philippe IV frappic apres 1637 & sacristic (?) \\
\hline 41 & Double tournois, Axe L.Gire- & & sacristie (?) \\
\hline 42 & Denier tournois, Dijion & 15819 ? & sacristie (?) \\
\hline & $\begin{array}{l}\text { Deux Detierer poins, Dole } \\
\text { Carolus au lion, Dole }\end{array}$ & $162(2 ?)$ & $\begin{array}{l}\text { satristie(?) } \\
\text { sacristie(?) }\end{array}$ \\
\hline
\end{tabular}

Fig. 8 - Planches des monnaies (P. Haut).

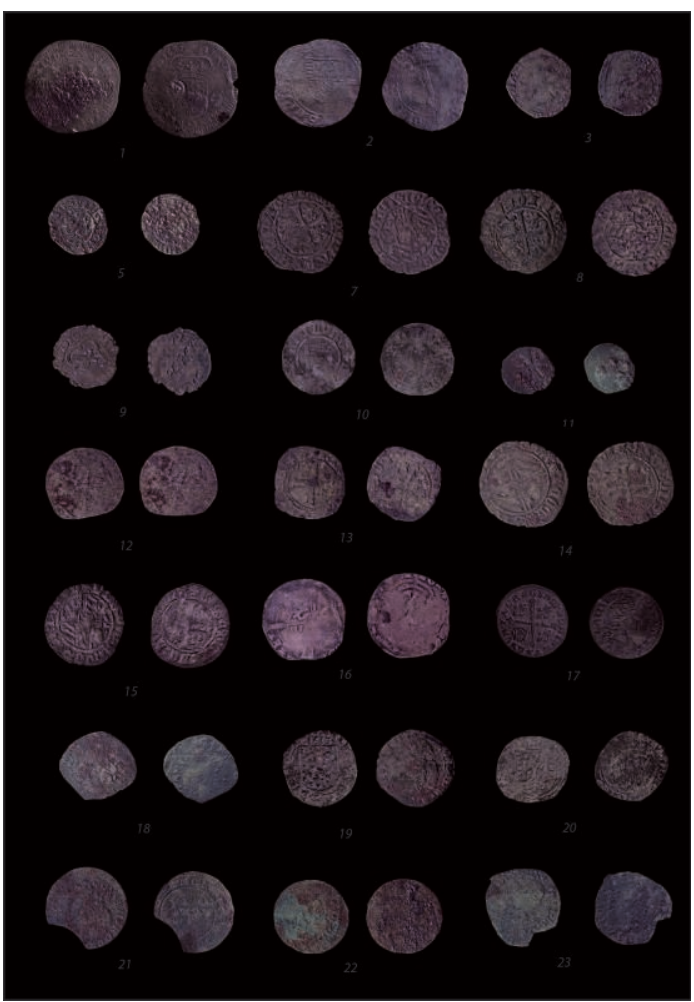

Reçu : 16 juillet 2012 - Accepté : 9 novembre 2012 


\section{NOTES}

1. D. BILLoIn, avec la collaboration de T. EUVRARD et V. LAMY, Domprel (Doubs). Cimetière communal. L'ancienne église Saint-Martin, Inrap, septembre 2010, 95 p.

2. J. Courtieu (dir.), Dictionnaire des communes du département du Doubs, Besançon, 1984, t.3, p. 1084-1090.

3. J. GAUTHIER, Répertoire archéologique du canton de Pierrefontaine (Doubs), Besançon, 1887, p. 3.

4. Besançon, Archives départementales du Doubs, série E 403/1-2.

5. Identification réalisée par le $\mathrm{D}^{\mathrm{r}}$ Nicolas Hubert, médecin légiste au Laboratoire d'anatomie de l'université de Besançon, que nous remercions vivement.

\section{INDEX}

Index géographique : France/Saint-Martin de Domprel (Doubs)

Mots-clés : sépulture, vestige

\section{AUTEURS}

DAVID BILLOIN

INRAP/Artehis-UMR 6298 